\title{
Motivos, personalidad y bienestar subjetivo en el voluntariado
}

\author{
Marcial E. Cabrera-Darias y Rosario J. Marrero-Quevedo*
}

Universidad de La Laguna (España).

\begin{abstract}
Resumen: El principal objetivo de este estudio fue analizar las relaciones entre las motivaciones del individuo para participar en actividades de voluntariado, los rasgos de personalidad y el bienestar subjetivo. Participaron 153 adultos, de edades entre los 17 y 65 años, que cumplimentaron las distintas medidas tanto on-line como en papel y lápiz. Los resultados mostraron que las motivaciones previas diferían entre los dos grupos de voluntarios. Los análisis correlacionales indicaron que algunos motivos como petición de la organización, circunstancias personales o experiencias vividas se asociaban a satisfacción vital y a emociones negativas en los voluntarios on-line; mientras que hacer algo útil o sentimientos de paz eran los motivos asociados a estos indicadores de bienestar para los voluntarios de la prueba en papel. Los rasgos de personalidad, principalmente el neuroticismo, mostraron relaciones más consistentes con el bienestar que las motivaciones en ambos grupos de voluntarios. Los análisis de regresión indicaron que las facetas de extraversión, como el gregarismo, la calidez o la actividad, fueron los predictores más potentes del bienestar de los voluntarios que hicieron las pruebas en papel; mientras que la disciplina, faceta de la responsabilidad, y la estabilidad emocional predecían el bienestar de los voluntarios on-line. Estos hallazgos sugieren que existen distintos perfiles de voluntarios que podrían estar determinando su participación en diversas actividades o colectivos. Además, las características personales parecen tener un mayor efecto en el bienestar que las motivaciones.

Palabras clave: trabajo voluntario, bienestar subjetivo, felicidad, facetas de personalidad.
\end{abstract}

\section{Introducción}

La conducta de ayuda comienza a estudiarse de forma sistemática a partir de los años 70 cuando surgen los primeros modelos que explican la toma de decisión de los individuos para ofrecer ayuda ante situaciones de emergencia (Latané y Darley, 1970). Este tipo de comportamiento se producía de manera puntual ante situaciones concretas y se asoció en cierta manera al altruismo de los individuos. Sin embargo, el voluntariado supone algo más que conductas de ayuda puntuales y una preocupación desinteresada por los demás (Myers, 1995), implica una cierta organización en actividades relevantes para la sociedad que en muchas ocasiones responden a intereses o motivaciones personales (Yubero y Larrañaga, 2002). De modo que, la persona voluntaria es aquella que desarrolla diversas actividades de manera libre, altruista y solidaria, ofrece su tiempo y facultades en instituciones formalmente constituidas que llevan a cabo distintos proyectos y no recibe ningún tipo de remuneración económica por su servicio (Ley 6/ 1996, BOE núm. 15).

La investigación acerca del voluntariado se ha centrado principalmente en identificar las variables sociodemográficas, actitudinales, personales o situacionales que caracterizan a

* Dirección para correspondencia [Correspondence address]:

Rosario J. Marrero Quevedo. Dpto. de Psicología Clínica, Psicobiología y Metodología. Facultad de Ciencias de la Salud. Sección de Psicología. Universidad de La Laguna. Campus de Guajara, s/n. 38205 La Laguna, Tenerife (España). E-mail: rmarrero@ull.edu.es
Title: Motives, personality and subjective well-being in volunteering.

Abstract: In this study, the aim was to analyze the relationships between individuals' motivation for to participate in volunteering, personality traits and subjective well-being. Data collection was conducted through paperand-pencil and on-line tests. A total of 153 adults, aged 17-65 years old, completed the instruments. The results showed that previous motivations differed between the two groups of volunteers. Correlational analyzes indicated that motivations, as requested by the organization, personal circumstances or life experiences, were associated with life satisfaction and negative affect in on-line volunteers; whereas that to do something useful or feelings of peace were the motives associated to well-being for volunteers who completed the test in paper and pencil. Personality traits, mainly neuroticism, showed more consistent relationships with well-being that the motivations in both groups of volunteers. Regression analyzes indicated that the facets of extraversion, gregariousness, warmth and activity were the strongest predictors of well-being for the volunteers who made the tests on paper and pencil; whereas that the facet of conscientiousness discipline and the emotional stability predicted well-being for the volunteers who completed the test on-line. These findings suggest that there are different profiles of volunteers that could be determining their participation in various activities or collectives. In addition, personal traits have shown to have a greater effect on well-being that the motivations to participate in volunteering.

Key words: volunteer work, subjective well-being, happiness, personality facets.

las personas que se involucran en estos servicios. Los primeros trabajos explicaban la participación voluntaria en base a características sociodemográficas (Cnaan y Cascio, 1998; Smith, 1994) proponiendo que las personas voluntarias serían aquellas que poseyeran características valoradas socialmente (Babchuk y Gordon, 1962; Wandersman, Florin, Friedmann y Meier, 1987). Se ha encontrado que las mujeres de mediana edad, casadas, con estudios superiores y con hijos en edad escolar (Hettman y Jenkins, 1990), así como personas que residen en la misma zona desde hace tiempo, que tienen una amplia red de amistades o que pertenecen a grupos formales, que no padecen ninguna enfermedad destacable, que están laboralmente activas, con prestigio profesional, ingresos elevados y un nivel cultural alto participan en actividades de voluntariado en mayor medida (Cnaan y Cascio, 1998; Smith, 1994; Thoits y Hewitt, 2001).

Por otra parte, se han estudiado las variables motivacionales o actitudinales que inciden en el voluntariado identificando motivaciones diversas. Clary et al. (1998) clasificaron los motivos en seis categorías según su función: comprensiva -aprender de la experiencia así como aplicar conocimientos y habilidades; desarrollo de valores -expresar el altruismo u otros valores humanitarios; de automejora -crecer y desarrollarse personalmente-; protectora -reducir la culpa por ser más afortunado que los otros; social -oportunidad para hacer amigos o implicarse en actividades que son valoradas positivamente por los otros; y profesional -mantener las habilidades adquiridas tras la formación. En un estudio realizado en España se encontró que la motivación más frecuente era 
la expresión de valores junto con el interés por la actividad, el compromiso organizacional, el cambio social, la religiosidad o el desarrollo personal (Chacón, Pérez, Flores y Vecina, 2010). Aunque coexisten varias motivaciones que pueden explicar la decisión de ser voluntario, ayudar a los demás, el deseo de promover las relaciones sociales y de obtener aprobación social parecen tener mayor peso (Metzer, 2003). Además, algunos estudios indican que la edad repercute en las principales motivaciones de los individuos para desarrollar la actividad voluntaria. Así, los jóvenes parecen tener más motivaciones relacionadas con la comprensión y la profesión mientras que los mayores una motivación de tipo social (Okun y Schultz, 2003). Estos resultados se confirman en otros grupos culturales, la edad guarda una relación directa con los motivos sociales y de valores y una relación inversa con los motivos profesionales (Ho, You y Fung, 2012). También estos autores encontraron que los motivos protectores incidían en la satisfacción vital de los voluntarios, aunque para las personas mayores en menor medida que para las de mediana edad o las jóvenes.

Los estudios que analizan el papel que juega la personalidad en la participación voluntaria indican que los voluntarios presentan altas puntuaciones en responsabilidad social y sociabilidad (Benson et al., 1980; Pierrucci y Noel, 1980); en imaginación y autoconfianza (Spitz y MacKinnon, 1993); en seguridad, flexibilidad y perseverancia (Herman y Usita, 1994) e informan de alta autoestima, estabilidad emocional y locus de control interno (Allen y Rushton, 1983). Elshaug y Metzer (2001), comparando a voluntarios con personas que ejercían una actividad similar pero remunerada, encontraron que los voluntarios se caracterizaban por una mayor extraversión y amabilidad. Otros estudios señalan que la socialización en actitudes o el desarrollo de una personalidad prosocial, como la empatía y ser servicial, son características importantes para la participación en voluntariado (Janoski, Musick y Wilson, 1998; Penner, 2002). De hecho, la empatía ha sido encontrada como antecedente de la ayuda en situaciones muy variadas (Eisenberg y Miller, 1987; Unger y Thumuluri, 1997). Clary y Miller (1986) han hallado que, entre otros factores, el altruismo expresado y puesto en práctica por los progenitores durante la infancia de sus hijos ejerce un efecto beneficioso en el altruismo de los hijos al llegar a adultos y repercute positivamente en el voluntario. Según Dulin, Hill y Anderson (2001) solo aquellas personas que se implican en servicios a la comunidad por razones altruistas experimentarían mayor bienestar.

Por otra parte, el bienestar y la satisfacción vital han mostrado tener una vinculación bidireccional con el voluntariado. Por un lado, la participación voluntaria puede modular los niveles experimentados de satisfacción vital (Dávila, 2003) pero además estar satisfecho puede incidir en la decisión de ser voluntario y en el mantenimiento de esta colaboración (Meier y Stutzer, 2008; Thoits y Hewitt, 2001). Los resultados muestran que la frecuencia con que una persona emplea tiempo en actividades voluntarias se relaciona positivamente con bienestar social (Dávila y Díaz, 2005; Harlow y
Cantor, 1996), apareciendo una mayor satisfacción vital y menor sintomatología depresiva en las personas mayores que dedican más horas al voluntariado (Jirovec y Hyduk, 1998; Wheeler, Gorey y Greenblatt, 1998). Thoits y Hewitt (2001) encuentran que la labor de voluntariado incide en la autoestima, el sentido de control, la salud física, la depresión, la satisfacción vital y la felicidad y que las personas que muestran mayor bienestar van a invertir más horas en las organizaciones. Además, el voluntario que tiene una motivación intrínseca por ayudar a los demás muestra mayor satisfacción vital, ya que ve cubiertas sus necesidades básicas de relación, autonomía y competencia (Kwok, Chui y Wong, 2012).

A pesar de los hallazgos encontrados, relativamente poca investigación se ha centrado en los beneficios psicológicos que las personas obtienen por implicarse en actividades voluntarias (Omoto, Snyder y Martino, 2000). De ahí que en este estudio un objetivo sea analizar las motivaciones de los individuos para ejercer el voluntariado y si éstas se relacionan de modo diferencial con el bienestar subjetivo, entendido en base a sus dos componentes, afectivo y cognitivo (Diener, 1984). A nivel afectivo se analizan las emociones positivas y emociones negativas, así como la felicidad que ha sido conceptualizada como el balance entre ambos tipos de emociones (Bradburn, 1969). A nivel cognitivo, se incluye la satisfacción con la vida que consiste en la valoración global que hace el individuo de sus logros de acuerdo a estándares previos subjetivos (Diener, Suh, Lucas y Smith, 1999). Además, un segundo objetivo es analizar conjuntamente las características personales de los voluntarios y las motivaciones para identificar la relevancia que puedan tener en su bienestar. La investigación previa revela que características como el Neuroticismo, la Extraversión o la Responsabilidad inciden en el bienestar de los individuos (DeNeve y Cooper, 1998; Marrero y Carballeira, 2011; Steel, Schmidt y Shultz, 2008). En personas mayores voluntarias se ha encontrado que la alta motivación acompañada de altas puntuaciones en Neuroticismo predicen un bajo bienestar (Pushkar, Reis y Morros, 2002) pero apenas existen estudios que analicen esta relación.

\section{Método}

\section{Participantes}

En este estudio han participado 153 personas que desarrollaban algún tipo de actividad en Organizaciones No Gubernamentales, de manera no remunerada, y que accedieron a participar en la investigación de manera voluntaria y no gratificada. El $71.1 \%$ de los participantes son mujeres, frente al $28.9 \%$ de hombres. La edad media es de 36.2 años, oscilando entre los 17 y los 65 años $(D T=12.02)$. El 44.7\% son personas solteras, el $23 \%$ casadas, el resto separadas o viudas y el $39.2 \%$ tiene entre 1 y 4 hijos. Respecto al nivel educativo, el $50.3 \%$ tiene nivel de estudios universitarios, seguido del $35.8 \%$ que tiene estudios de bachillerato o formación 
profesional, el $9.3 \%$ posee estudios primarios y un .7\% sólo sabe leer y escribir. De estos, sólo algo más de un tercio trabajan de modo remunerado $(37.8 \%)$, otro porcentaje similar está en situación de desempleo (38.5\%), el $19.6 \%$ son estudiantes y un $4.1 \%$ son jubilados.

Entre los motivos que declaran los participantes para haberse implicado en su momento en labores de voluntariado, se encuentran en primer lugar "ayudar a otros" (60\%), seguido de "realización personal" (56.9\%), "por la necesidad de hacer algo útil" (51.6\%), por la "oportunidad de relaciones profesionales y de aprendizaje" (31.3\%) y un $18.8 \%$ alude a la "oportunidad de relaciones sociales".

Los participantes del estudio llevaban entre 1 mes y 30 años colaborando como voluntarios ( $M=44.52$ meses; $D T$ $=65.30)$. Concretamente el $17.4 \%$ llevaba menos de 6 meses; el $18.1 \%$ entre uno y dos años; otro $18.8 \%$ entre tres y cinco años; y un $12.2 \%$ más de 10 años. Por otra parte, la media de horas semanales dedicadas al voluntariado fue de $5.68(D T=6.86)$, aunque un $40 \%$ dedicaba al menos 4 horas a tal actividad, el $19.3 \%$ empleaba entre 8 y $15 \mathrm{~h}$. y el $7.6 \%$ de 20 a 40 h.

\section{Instrumentos}

Entrevista semiestructurada de variables sociodemográficas (Marrero, Carballeira y Rodríguez, 2007). Se diseñó para recoger datos, tales como el género, la edad, el nivel de estudios, estado civil, entre otros. Además, se incluyó un listado de 19 motivos por los que los participantes desarrollaban la actividad de voluntariado con formato multi-respuesta donde debían señalar todos aquellos motivos que se aplicaban a su caso (p ej: "por realización personal"; "por ayudar a otros"; "por petición directa desde la organización"; "porque me siento bien, en paz. conmigo mismo/a"; "por diversión"; "por convicción religiosa" o "porque dispongo de tiempo libre") identificando de este modo si cada uno de esos motivos estaba presente o ausente. Por otro lado, se registró el tiempo en meses desde que comenzó a desarrollar dicha labor y el número de horas semanales destinadas a la misma.

Inventario de Personalidad Revisado -NEO-PI-R- (Costa y McCrae, 1992). Se empleó la versión española de Cordero, Pamos y Seisdedos (1999). Este instrumento mide a través de 240 ítems, que se puntúan en una escala que va desde 0 a 4 puntos, los cinco grandes factores de personalidad: Extraversión, Neuroticismo, Apertura a la experiencia, Amabilidad y Responsabilidad, así como sus 30 facetas, seis para cada uno de los grandes rasgos. Una alta puntuación implica poseer en gran medida el rasgo evaluado. En la validación del inventario la consistencia interna de las escalas oscilaba entre .89 y .95 . En este estudio la consistencia interna osciló de .80 a .88 y para las facetas de .33 a .78 .

Satisfaction with Life Scale -SWLS- (Diener, Emmons, Larsen y Griffin, 1985). La prueba fue traducida al español por el equipo investigador y evalúa, a través de 5 ítems, el juicio cognitivo del individuo acerca de la satisfacción global con su propia vida, comparando sus circunstancias vitales con un estándar particular. Cada ítem se responde en una escala de 1 (nada satisfecho) a 7 (muy satisfecho). La consistencia interna de la escala original fue de .87 y la fiabilidad test-retest .82. La versión española con adolescentes alcanzó un alpha de Cronbach de .84 (Atienza, Pons, Balaguer y García-Merita, 2000) y .88 con una muestra amplia de adultos (Vázquez, Duque y Hervás, 2013). En este estudio, con una traducción equivalente, se obtuvo una consistencia interna de .88 .

Positive and Negative Affect Schedules -PANAS- (Watson, Clark y Tellegen, 1988). El instrumento evalúa, en una escala de 0 a 7 puntos, las emociones positivas y las emociones negativas presentes en el momento de contestar la prueba. Consta de 10 calificativos positivos y otros 10 negativos. Las escalas, en la versión original de la prueba, mostraron una frabilidad adecuada de .88 para emociones positivas y $.85 \mathrm{pa}-$ ra las negativas. La validación con población española arrojó la misma estructura bidimensional, presentando las escalas una consistencia interna de .90 para emociones positivas y .88 para emociones negativas (Sandín, Chorot, Lostao, Joiner, Santed y Valiente, 1999). En este estudio se empleó la versión traducida por el equipo de investigación, que se ajusta en gran medida a la empleada en la validación española, mostrando las escalas una consistencia de .84 para emociones positivas y .90 para emociones negativas.

La Subjective Happiness Scale -SHS- (Lyubomirsky y Lepper, 1999) es una escala que mide la felicidad autoevaluada. El individuo a través de 4 ítems, con un rango de respuesta de 1 a 7 , señala si se siente en general nada o muy feliz en su vida en función de estándares personales o de procesos de comparación social. La escala original fue validada a través de 14 muestras diferentes, tanto de estudiantes como de adultos mayores, obteniendo una fiabilidad entre .79 y .94. La fiabilidad test-retest a las tres semanas oscilaba entre $.55 \mathrm{y}$ .90. En España no se han llevado a cabo estudios de validación de esta escala, aunque Extremera, Salguero y Fernández-Berrocal (2011) informan de un alpha de .80 y .79, transcurrido un período de siete semanas. En este estudio la versión traducida obtuvo una consistencia interna de .51.

\section{Procedimiento}

Con el objetivo de tener una mayor representatividad del colectivo de voluntariado se identificaron a través de la web las distintas ONG's de la Isla de Tenerife. A continuación, se procedió a contactar con la directiva de cada una de ellas vía e-mail, explicándole los objetivos de la investigación y pidiendo su colaboración. De esta manera, la directiva se encargó de hacer llegar la información al voluntariado junto con un enlace en el que podían encontrar la batería de pruebas en formato on-line. Aunque también se administró a un $29.4 \%$ de los participantes, que así lo solicitaron, las pruebas en formato papel. La cumplimentación de las mismas supuso una duración aproximada de 50 minutos. En todos los casos se explicaba por escrito los objetivos de la investigación aclarando que la cumplimentación de las pruebas supo- 
nía el consentimiento expreso para emplear la información con fines investigadores.

El análisis de los datos se realizó con el programa estadístico SPSS (Versión 19). En un primer momento, se observó la distribución de la muestra en función de variables sociodemográficas y de los motivos que aludían las personas para su participación en actividades de voluntariado. Seguidamente se analizó, a través de un ANOVA, si había diferencias en bienestar en función de que estuviera presente o ausente cada uno de los motivos. También se aplicó un ANOVA para comprobar si los voluntarios que habían cumplimentado las pruebas en papel y los que las habían hecho on-line se diferenciaban en características sociodemográficas y en el resto de las medidas evaluadas. Mediante correlación de Pearson y de Spearman se analizó la relación entre variables sociodemográficas, motivaciones para dedicarse al voluntariado, personalidad y bienestar subjetivo en ambos grupos de voluntarios. Por último, se aplicó análisis de regresión múltiple, con el método paso a paso, con el objeto de identificar las características sociodemográficas, de personalidad y motivos que incidían en el bienestar de ambos grupos de personas (evaluados on-line frente a evaluados en formato papel) que desarrollaban labores voluntarias. Una vez identificados los rasgos amplios que explicaban cada uno de los indicadores de bienestar, se seleccionaron las facetas de aquellos rasgos que resultaron significativos en cada caso y junto con los motivos y variables sociodemográficas que también hubieran mostrado su capacidad predictiva en los análisis anteriores se aplicaron nuevos análisis de regresión.

\section{Resultados}

Se analizó a través de un ANOVA si había diferencias en bienestar en función de que estuviera presente o ausente cada uno de los motivos que aludían los voluntarios. Únicamente aparecieron diferencias significativas en 3 de los 19 motivos. Los voluntarios con la motivación de petición por parte de la organización mostraron mayor felicidad que los que no tenían este tipo de motivación $[F(1,104)=4.57, p=$ .035] y los que mencionaron las circunstancias personales $[F(1,105)=9.83, p=.002]$ y las experiencias vividas $[F(1,105)=4.47, p=.037]$ mostraron más emociones negativas que los que no señalaron estos motivos.

Dado que del total de participantes hubo personas que cumplimentaron las pruebas on-line y otras a través de un cuestionario de papel y lápiz se analizó si había diferencias entre ambos grupos en las distintas variables evaluadas. Los resultados del ANOVA mostraron que no había diferencias en ninguna de las variables sociodemográficas consideradas, ni en el tiempo, ni horas que dedicaban al voluntariado: gé- nero $[F(1,140)=1.40, p=.239]$, edad $[F(1,140)=1.46, p=$ $.229]$, estado civil $[F(1,140)=.74, p=.390]$, nivel de estudios $[F(1,140)=1.31, p=.289]$, tiempo que lleva ejerciendo el voluntariado $[F(1,140)=1.51, p=.221]$ y horas semanales empleadas $[F(1,140)=.81, p=.776]$. Tampoco se encontraron diferencias significativas entre los grupos en personalidad: neuroticismo $[F(1,103)=.42, p=.519]$, extraversión $[F(1,103)=1.02, p=.314]$, apertura a la experiencia $[F(1,103)=.06, p=.805]$, amabilidad $[F(1,103)=.70, p=$ $.404]$ y responsabilidad $[F(1,103)=1.59, p=.211]$. En las medidas de bienestar los participantes que cumplimentaron las pruebas en papel mostraron más emociones positivas que los del grupo on-line $[F(1,106)=5.15, p=.025]$ pero no se encontraron diferencias en felicidad $[F(1,105)=1.51, p=$ $.222]$, satisfacción vital $[F(1,108)=.26, p=.612]$ o emociones negativas $[F(1,106)=.29, p=.593]$.

En cuanto a la motivación subyacente para dedicarse al voluntariado se encontraron diferencias entre los dos grupos de participantes en la mayor parte de los motivos. Los voluntarios que cumplimentaron la prueba en papel señalaron con mayor frecuencia los motivos de realización personal $(73.3 \%)$, circunstancia personal $(60 \%)$, petición de la organización $(57.8 \%)$ y estudios afines $(55.6 \%)$; mientras que los que la hicieron on-line indicaron principalmente ayudar a otros $(67.3 \%)$, sentimiento de paz $(60.7 \%)$ o hacer algo útil $(55.1 \%)$. El ANOVA en que se compararon las motivaciones de ambos grupos indicaron que los voluntarios de la prueba en papel puntuaron más alto en los motivos de petición de la organización $[F(1,150)=65.41, p<.000]$, convicción religiosa $[F(1,150)=52.62, p<.000]$, jubilación $[F(1$, $150)=45.38, p=.000]$, conocer mejor la temática $[F(1,150)$ $=5.02, p=.027]$, estudios afines $[F(1,150)=6.52, p=.012]$, circunstancia personal $[F(1,150)=66.41, p<.000]$ y realización personal $[F(1,150)=6.99, p=.009]$; mientras que los que cumplimentaron la prueba on-line informaron de motivaciones relacionadas con sentimientos de paz $[F(1,150)=$ $34.78, p=.000]$, diversión $[F(1,150)=10.55, p=.001]$, disponer de tiempo $[F(1,150)=29.84, p<.000]$, devolver algo a la sociedad $[F(1,150)=7.92, p=.006]$, ayudar a otros $[F(1,150)=7.15, p=.008]$ y por experiencias vividas $[F(1$, $150)=15.74, p<.000]$.

Teniendo en cuenta que los motivos que estaban a la base de la dedicación al voluntariado variaban en ambos subgrupos de voluntarios el resto de análisis se llevaron a cabo para cada grupo por separado. El análisis correlacional entre los motivos, las variables sociodemográficas y los indicadores de bienestar indicaba que en ambos grupos los motivos estaban más relacionados con características sociodemográficas que con el bienestar (Véase Tabla 1). 
Tabla 1. Correlación entre variables sociodemográficas, con los motivos y el bienestar del voluntariado.

\begin{tabular}{|c|c|c|c|c|c|c|c|c|c|c|c|c|c|c|c|c|}
\hline \multirow[t]{2}{*}{ Variables } & \multicolumn{2}{|c|}{ Género } & \multicolumn{2}{|c|}{ Edad } & \multicolumn{2}{|c|}{ Estado Civil } & \multicolumn{2}{|c|}{$\begin{array}{l}\text { Nivel de } \\
\text { estudios }\end{array}$} & \multicolumn{2}{|c|}{ Felicidad } & \multicolumn{2}{|c|}{$\begin{array}{c}\text { Satisfacción } \\
\text { Vital }\end{array}$} & \multicolumn{2}{|c|}{$\begin{array}{c}\text { Emociones } \\
\text { positivas }\end{array}$} & \multicolumn{2}{|c|}{$\begin{array}{c}\text { Emociones } \\
\text { negativas }\end{array}$} \\
\hline & Papel & $\begin{array}{l}\text { On- } \\
\text { line }\end{array}$ & Papel & $\begin{array}{l}\text { On- } \\
\text { line }\end{array}$ & Papel & $\begin{array}{l}\text { On- } \\
\text { line }\end{array}$ & Papel & $\begin{array}{l}\text { On- } \\
\text { line }\end{array}$ & Papel & $\begin{array}{l}\text { On- } \\
\text { line }\end{array}$ & Papel & $\begin{array}{l}\text { On- } \\
\text { line }\end{array}$ & Papel & On-line & Papel & $\begin{array}{l}\text { On- } \\
\text { line }\end{array}$ \\
\hline Influencia de conocido & .12 & -.15 & .16 & .05 & .12 & -.00 & -.26 & -.09 & .15 & -.04 & .02 & -.05 & .10 & -.01 & -.01 & .10 \\
\hline Familiar voluntario & -.12 & -.17 & -.28 & -.15 & -.19 & $-.21 *$ & -.09 & -.09 & -.23 & -.09 & $-.46^{* *}$ & .07 & -.15 & -.04 & .14 & .17 \\
\hline Conocido voluntario & .19 & $-.21 *$ & $.31 *$ & -.09 & .16 & .04 & .14 & -.09 & .15 & -.16 & .13 & -.05 & -.03 & -.04 & -.08 & .06 \\
\hline Petición organización & .02 & .09 & .01 & -.14 & .07 & -.04 & .15 & $.24^{*}$ & .16 & .20 & .03 & $.23^{*}$ & .05 & .09 & -.06 & -.11 \\
\hline Sentimiento de paz & .16 & .10 & $.41^{* *}$ & .11 & .12 & .12 & .03 & -.04 & .19 & .17 & .13 & .17 & .21 & .17 & $-.40 *$ & .05 \\
\hline Obligación moral & -.09 & -.08 & .20 & .09 & .09 & -.11 & -.08 & -.08 & .07 & -.01 & .06 & -.08 & .10 & .02 & .04 & -.01 \\
\hline Diversión & -.20 & .08 & .14 & $-.22 *$ & .01 & .01 & .13 & .11 & .10 & .11 & .17 & .11 & .07 & .18 & -.04 & .01 \\
\hline Convicción religiosa & .01 & .01 & -.06 & -.17 & -.09 & $-.19 *$ & -.15 & .17 & -.20 & .17 & -.28 & .10 & -.29 & .02 & -.22 & .00 \\
\hline Disponer de tiempo & - & .15 & - & -.05 & - & -.14 & - & .01 & - & .11 & - & .10 & - & .02 & - & -.12 \\
\hline Jubilación & $.33^{*}$ & .06 & .11 & .14 & -.04 & .03 & .27 & .08 & -.06 & .22 & -.09 & .12 & -.04 & -.12 & -.07 & -.15 \\
\hline Conocer mejor el tema & $.39 * *$ & $-.22 *$ & $-.31 *$ & -.03 & -.26 & -.12 & .11 & .03 & -.24 & .07 & -.03 & .06 & -.15 & .16 & -.16 & -.04 \\
\hline Estudios afines & .08 & .02 & .26 & -.18 & .12 & -.07 & .16 & $.33^{* * *}$ & -.12 & .04 & -.26 & .19 & -.03 & -.01 & .21 & $-.30^{* *}$ \\
\hline Hacer algo útil & .01 & .07 & .08 & $.21 *$ & -.04 & -.01 & .17 & -.00 & .10 & .03 & $.36^{*}$ & -.11 & .15 & -.06 & -.04 & -.02 \\
\hline Apoyar organización & .04 & -.05 & -.02 & $-.22 *$ & -.19 & -.13 & .16 & -.08 & .07 & -.03 & .06 & .05 & .17 & .04 & -.13 & .15 \\
\hline Devolver a la sociedad & .26 & -.08 & .18 & .10 & -.00 & -.02 & .15 & .14 & .04 & -.00 & -.10 & -.00 & .31 & .05 & -.15 & .06 \\
\hline Circunstancia personal & .06 & -.13 & -.23 & -.05 & -.23 & -.01 & .12 & -.13 & -.20 & -.04 & -.06 & -.13 & -.11 & -.21 & .14 & $.46^{* * *}$ \\
\hline Realización personal & .18 & .05 & -.05 & -.10 & .05 & .12 & .07 & -.04 & .17 & .10 & .09 & .07 & -.00 & .09 & -.16 & .08 \\
\hline Ayudar a otros & .01 & .14 & -.03 & .11 & .01 & .00 & -.00 & .02 & -.22 & -.06 & -.17 & -.11 & -.27 & -.00 & .03 & .06 \\
\hline Experiencias vividas & - & $-22^{*}$ & - & .05 & - & .10 & - & -.15 & - & .11 & - & -.09 & - & .11 & - & $.27 *$ \\
\hline Tiempo voluntario & $-44 * *$ & .11 & .27 & $.38^{* * *}$ & .25 & $.33 * * *$ & .02 & .09 & .15 & .05 & .10 & .09 & .05 & .17 & .05 & .01 \\
\hline Horas semana voluntario & -.13 & .19 & .17 & .05 & .16 & .17 & -.20 & .02 & .06 & .12 & .27 & .07 & .29 & .08 & -.23 & .04 \\
\hline
\end{tabular}

Así, para las personas que cumplimentaron la prueba en papel, el género y la edad se asociaban a motivos como jubilación, conocer mejor la temática, tener un conocido voluntario o sentimientos de paz. Sólo tener un familiar voluntario, hacer algo útil y el sentimiento de paz se relacionaron con algún indicador de bienestar. En el caso de las personas que rellenaban la prueba on-line, el género se relacionaba con motivos como tener un conocido voluntario o conocer el tema y la edad se asociaba negativamente a diversión y positivamente a hacer algo útil. Los motivos relacionados con petición de la organización, experiencias vividas, circunstancias personales y tener estudios afines se asociaron a algún indicador de bienestar. Sin embargo, ni el tiempo ni las horas semanales dedicadas al voluntariado se relacionaron con el bienestar.

Las características de personalidad se asociaron en mayor medida al bienestar subjetivo que los motivos en ambos grupos de participantes, aunque aparecía un patrón de relación ligeramente distinto (Véase Tabla 2).

Tabla 2. Correlación entre características de personalidad y bienestar subjetivo.

\begin{tabular}{|c|c|c|c|c|c|c|c|c|}
\hline \multirow[t]{2}{*}{ Variables } & \multicolumn{2}{|c|}{ Felicidad } & \multicolumn{2}{|c|}{ Satisfacción vital } & \multicolumn{2}{|c|}{ Emociones positivas } & \multicolumn{2}{|c|}{ Emociones Negativas } \\
\hline & Papel & On-line & Papel & On-line & Papel & On-line & Papel & On-line \\
\hline Neuroticismo & $-.46^{* *}$ & $-.31 * *$ & $-.53 * *$ & $-.39 * * *$ & $-.60 * * *$ & $-.49 * * *$ & $.81^{* * *}$ & $.75^{* * *}$ \\
\hline Extraversión & $.55^{* *}$ & .22 & $.36^{*}$ & .19 & $.63 * * *$ & $.57 * * *$ & $-.59 * * *$ & -.23 \\
\hline Apertura & .06 & .14 & .10 & .03 & .11 & $.39 * * *$ & -.25 & -.15 \\
\hline Amabilidad & .20 & .01 & .40 & -.19 & $.43^{*}$ & -.13 & -.36 & -.11 \\
\hline Responsabilidad & .25 & $.48^{* * *}$ & $.50 * *$ & $.28 *$ & .21 & $.45^{* * *}$ & -.16 & -.52 \\
\hline
\end{tabular}
${ }^{*} p<.05,{ }^{* *} p<.01, * * * p<.001$

El neuroticismo se relacionó negativamente con todos los indicadores de bienestar en ambos grupos. Sin embargo, la extraversión se asociaba al bienestar para el grupo de personas que hizo las pruebas en papel pero no para las que la hicieron on-line, para las cuales fue más relevante la responsabilidad. La amabilidad se relacionaba con satisfacción vital y emociones positivas para las personas que hicieron las pruebas en papel y la apertura a la experiencia se asociaba a las emociones positivas en las que la cumplimentaron on-line.

Con el propósito de analizar el peso que tenían las características sociodemográficas, el tiempo de voluntariado, los motivos y la personalidad en el bienestar se llevaron a cabo diversos análisis de regresión múltiple para cada grupo de voluntarios por separado. Se aplicó una regresión paso a paso en la que se incluyeron como variables predictoras en un primer bloque la edad y el género que habían mostrado tener relación con algunas motivaciones para ser voluntario y con ciertos indicadores de bienestar. En el segundo bloque se incluyó el tiempo que llevaban dedicados al voluntariado. En un tercer bloque se introdujeron los 7 motivos que habían mostrado asociaciones previas con alguno de los indicadores de bienestar. Y por último, en el cuarto bloque, se incluyeron los Cinco Grandes de Personalidad. En cada análisis la variable criterio era uno de los cuatro indicadores de bienestar subjetivo. Los análisis correlacionales previos mostraron que no existía multilcolinealidad entre las distintas variables 
predictoras. Las correlaciones de mayor magnitud se dieron entre neuroticismo y extraversión $(r=-.60)$, lo que indica que no comparten más de un 36\% de la varianza; o entre los motivos hacer algo útil y circunstancia personal $(r=.53)$, teniendo una varianza común del $28 \%$. Además, los indicadores de tolerancia y el factor de inflación de la varianza mostraron que no había colinealidad entre los predictores incluidos en cada ecuación. Los resultados de los distintos análisis de regresión indicaron que había diferencias en las variables que predecían el bienestar en ambos grupos de voluntarios. La felicidad dependía exclusivamente de la extraversión $(\beta=$ $.55, p<.01)$ para las personas que hicieron la prueba en papel $[F(1,24)=10.27, p<.01]$ explicando un $27 \%$ de la varianza; mientras que la responsabilidad $(\beta=.52, p<.001)$ explicaba el $26 \%$ de la felicidad para los que la rellenaron online $[F(1,65)=24.21, p<.001]$. La satisfacción vital de los voluntarios de cuestionarios en papel $[F(3,23)=10.28, p<$ .001] guardaba relación con la motivación de hacer algo útil $(\beta=.33, p<.05)$, con la responsabilidad $(\beta=.46, p<.01) \mathrm{y}$ la extraversión $(\beta=.36, p<.05)$, explicando el $51 \%$ de la varianza; mientras que para los voluntarios on-line $[F(3,63)=$ 24.21, $p<.001]$ se asociaba a la petición de la organización $(\beta=.22, p<.05)$, al neuroticismo $(\beta=-.39, p<.001)$ y negativamente a la amabilidad $(\beta=-.24, p<.05)$, explicando un $21 \%$ de la varianza. Para los voluntarios que cumplimentaron las pruebas en papel, la edad $(\beta=.43, p<.01)$ y la extraversión $(\beta=.58, p<.001)$ predijeron el $56 \%$ de la varianza de emociones positivas $[F(2,23)=16.91, p<.001]$; por otra parte la edad en sentido negativo $(\beta=-.10$, n.s.) y el neuroticismo $(\beta=.78, p<.001)$ entraron en la ecuación explicando el $68 \%$ de la varianza de emociones negativas $[F(2,23)=28, p<.001]$. En el caso de los voluntarios de las pruebas on-line, el $48 \%$ de las emociones positivas $[F(3,62)=$ 20.71, $p<.001]$ fueron predichas por neuroticismo $(\beta=-$ $.41, p<.001)$, extraversión $(\beta=.42, p<.001)$ y amabilidad $(\beta=-.20, p<.05)$; y el $67 \%$ de las emociones negativas $[F(2,63)=66.05, p<.001]$ fueron predichas por el motivo circunstancias personales $(\beta=.36, p<.001)$ y neuroticismo $(\beta=.62, p<.001)$.

En un siguiente momento, se analizó qué facetas de los Cinco Grandes eran las que tenían más capacidad predictiva del bienestar para ambos grupos de voluntarios. De modo que se aplicaron nuevamente análisis de regresión, incluyendo como variables predictoras los motivos que habían entrado a formar parte de la ecuación de regresión en los análisis anteriores, la edad y las facetas correspondientes a aquellos factores de personalidad que también habían mostrado su capacidad predictiva (Véase Tabla 3).

Tabla 3. Análisis de regresión de las facetas de personalidad sobre medidas de bienestar subjetivo.

\begin{tabular}{|c|c|c|c|c|c|c|c|c|c|c|c|c|c|c|c|c|}
\hline \multirow[b]{2}{*}{ Variables } & \multicolumn{4}{|c|}{ Felicidad } & \multicolumn{4}{|c|}{ Satisfacción Vital } & \multicolumn{4}{|c|}{ Emociones Positivas } & \multicolumn{4}{|c|}{ Emociones Negativas } \\
\hline & $\beta$ & $\begin{array}{c}R^{2} \\
\text { ajust. }\end{array}$ & $\Delta R^{2}$ & $\mathrm{~F}$ & $\beta$ & $\begin{array}{c}R^{2} \\
\text { ajust. }\end{array}$ & $\Delta R^{2}$ & $\mathrm{~F}$ & $\beta$ & $\begin{array}{c}R^{2} \\
\text { ajust. }\end{array}$ & $\Delta \mathrm{R}^{2}$ & $\mathrm{~F}$ & $\beta$ & $\begin{array}{c}R^{2} \\
\text { ajust. }\end{array}$ & $\Delta R^{2}$ & $\mathrm{~F}$ \\
\hline \multicolumn{17}{|c|}{ Voluntarios pruebas papel } \\
\hline \multicolumn{17}{|c|}{ Paso 1} \\
\hline Gregarismo & $.60^{* * *}$ & .33 & .36 & $14.38^{* * *}$ & & & & & & & & & & & & \\
\hline Hacer algo útil & & & & & .27 & & .22 & $7.79 * *$ & & & & & & & & \\
\hline Edad & & & & & & & & & $.42^{* *}$ & & .24 & $8.48^{* *}$ & $-.30 *$ & & .26 & $9.35^{* *}$ \\
\hline \multicolumn{17}{|l|}{ Paso 2} \\
\hline Disciplina & & & & & $.53^{* *}$ & .41 & .24 & $11.24 * * *$ & & & & & & & & \\
\hline Calidez & & & & & & & & & $.38^{*}$ & & .26 & $13.24 * * *$ & & & & \\
\hline Depresión & & & & & & & & & & & & & $.51 * * *$ & & .38 & $22.66^{* * *}$ \\
\hline \multicolumn{17}{|l|}{ Paso 3} \\
\hline Actividad & & & & & & & & & $.31 *$ & .53 & .08 & $11.56^{* * *}$ & & & & \\
\hline Hostilidad & & & & & & & & & & & & & $.33^{*}$ & .68 & .08 & $21 * * *$ \\
\hline \multicolumn{17}{|c|}{ Voluntarios pruebas on-line } \\
\hline \multicolumn{17}{|l|}{ Paso 1} \\
\hline Disciplina & $.48^{* * *}$ & .22 & .23 & $21.16^{* * *}$ & & & & & & & & & & & & \\
\hline Petición organización & & & & & $.21 *$ & & .06 & $4.13^{*}$ & & & & & & & & \\
\hline Actividad & & & & & & & & & $.45^{* *}$ & & .33 & $32.15^{* * *}$ & & & & \\
\hline Circunstancia personal & & & & & & & & & & & & & $.32 * * *$ & & .33 & $33.84 * * *$ \\
\hline \multicolumn{17}{|l|}{ Paso 2} \\
\hline Depresión & & & & & $-.40 * * *$ & & .16 & $9.26 * * *$ & & & & & $.45^{* * *}$ & & .33 & $65.16^{* * *}$ \\
\hline Emocionalidad positiva & & & & & & & & & $.19^{*}$ & & .07 & $21.54 * * *$ & & & & \\
\hline \multicolumn{17}{|l|}{ Paso 3} \\
\hline Sensibilidad a los demás & & & & & $-.26^{*}$ & .25 & .07 & $8.72 * * *$ & & & & & & & & \\
\hline Ansiedad & & & & & & & & & $-.46 * * *$ & .58 & .20 & $31.38^{* * *}$ & $.24^{*}$ & .67 & .03 & $48.33^{* * *}$ \\
\hline
\end{tabular}

Ansiedad

${ }^{*} p<.05 ;{ }^{* *} p<.01 ; * * * p<.001$

En el modelo final, la felicidad fue predicha por el gregarismo $(\beta=.60, p<.001)$ para el grupo de voluntarios que hicieron la prueba en papel $[F(1,26)=14.38, p<.001]$ y por la disciplina $(\beta=.48, p<.001)$ para los que la realizaron online $[F(1,72)=21.16, p<.001]$. La satisfacción vital de los voluntarios de las pruebas en papel fue predicha por la disci- plina $(\beta=.53, p<.01)$ y aunque el motivo de hacer algo útil entró en la ecuación no resultó significativo, para los voluntarios on-line fue más relevante el motivo de petición de la organización $(\beta=.21, p<.01)$, la baja depresión $(\beta=-.40, p$ $<.001)$ y baja sensibilidad hacia los demás $(\beta=-.26, p<$ $.01)$. En cuanto a las emociones positivas y negativas de los 
voluntarios de pruebas en papel ambas fueron predichas por la edad aunque en sentido inverso y mientras las emociones positivas se asociaron a calidez $(\beta=.38, p<.05)$ y a actividad $(\beta=.31, p<.05)$, las negativas lo hicieron con depresión $(\beta=.51, p<.001)$ y hostilidad $(\beta=.33, p<.05)$. En los voluntarios on-line, las emociones positivas fueron explicadas por la actividad $(\beta=.45, p<.01)$, emocionalidad positiva $(\beta$ $=.19, p<.05)$ y baja ansiedad $(\beta=-.46, p<.001)$, mientras que las negativas se explicaron por la depresión $(\beta=.45, p<$ $.001)$, la ansiedad $(\beta=.24, p<.05)$ y por el motivo de circunstancias personales $(\beta=.32, p<.001)$.

\section{Discusión}

En este estudio se han analizado las características sociodemográficas, motivacionales y personales del voluntariado con el objetivo principal de identificar cuáles inciden en mayor medida en el bienestar de este colectivo. Aunque resulta difícil cuantificar la participación del voluntariado en las diversas ONGs debido a altas y bajas frecuentes, se estima que en España alrededor de un 18\% de las personas adultas dedican su tiempo a actividades de voluntariado social frente a la media europea que se sitúa en un 34\% (GHK, 2010). Sin embargo, según datos del Centro de Investigaciones Sociológicas (CIS, 2011), el 31\% dice haber realizado alguna vez este tipo de acciones. Por otra parte, se ha encontrado que algunas personas pierden la motivación por colaborar con estas asociaciones debido a discrepancias entre las expectativas iniciales y tareas asignadas, a problemas de comunicación con el equipo de trabajo, o a la falta de refuerzo del trabajo bien realizado, entre otros (Juventud en Acción, 2014). Incluso en algunos casos llegan a experimentar burnout debido a la escasa gratificación, lo que a su vez reduce la intención de continuar en la actividad voluntaria (Chacón, 2005; Chacón y Vecina, 1999). De modo que este trabajo resulta relevante en la medida en que permite identificar cuáles son las principales motivaciones y características personales de los individuos que informan de mayor bienestar. Por otra parte, podría emplearse por las ONGs, no sólo para hacer campañas de captación de voluntariado que muestre estas características, sino además para mantener su labor en el tiempo trabajando las distintas motivaciones implicadas.

Los resultados de este estudio indicaron que el voluntariado ha estado formado principalmente por mujeres, de edad media, con estudios universitarios, mayoritariamente solteras, algo más de un tercio tenía hijos y trabajaba de forma remunerada. Las encuestas sobre participación en organizaciones benéficas y de voluntariado en España señalan que entre un 57.18\% (INE, 2007) y un 66.54\% (FEAPS, 2011) son mujeres y cerca de tres cuartas partes tienen entre 18 y 30 años (Plataforma de Voluntariado de España, 2011, 2013). Investigaciones en otros países también encuentran un perfil similar al obtenido en nuestro estudio, apareciendo una mayor proporción de mujeres que de hombres, aunque señalan que gran parte están casadas y con hijos (Hettman y Jenkins, 1990; Plagnol y Huppert, 2010; Thoits y Hewitt,
2001). Hay que tener en cuenta que en España, en los últimos 35 años, el índice de matrimonios ha descendido y la edad media a la que se establece dicho vínculo ha aumentado (Instituto de la Mujer, 2013), lo que podría explicar la baja tasa de personas casadas y sin hijos.

El voluntariado que participó en este estudio además tuvo un perfil diferencial en cuanto a las motivaciones subyacentes según accediésemos a ellos a través de la red o mediante contacto directo. Algunos estudios han señalado que las personas que cumplimentan pruebas on-line y las que lo hacen en papel pueden mostrar características distintas (Buchanan et al., 2005). Así, en el voluntariado que cumplimentó las pruebas en papel se encontró que los motivos de jubilación o conocer mejor la temática estuvieron más presentes en las mujeres que en los hombres; las personas de más edad mostraron motivaciones relacionadas con el sentimiento de paz o tenían personas conocidas en el voluntariado, mientras que para los jóvenes era más importante querer conocer mejor la temática. En el grupo de voluntarios que participaron on-line, a mayor edad aparecían más motivos relacionados con hacer algo útil, mientras que a menor edad más motivos relacionados con la diversión y con apoyar a la organización. Estos resultados coinciden parcialmente con estudios previos que encuentran que la edad está inversamente relacionada con motivos relacionados con comprender mejor el servicio que se ofrece o con la carrera profesional y positivamente con las relaciones sociales (Okun y Schultz, 2003). Además, en los voluntarios on-line tener mayor cualificación académica se asoció a estudios afines y a petición de la organización. Estos resultados podrían explicarse en función de esa necesidad de las personas jóvenes de vincularse a actividades que les permitan tener un mayor conocimiento profesional (Okun y Schultz, 2003). Por otra parte, el hecho de que la organización cuente con ellos permitirá que se mantenga el compromiso a través de ese reconocimiento social (Cnaan y Cascio, 1998)

En nuestro estudio, los motivos que subyacían a la decisión de ser voluntario para las personas que contestaron en papel fueron principalmente motivaciones autocentradas, como la realización personal o circunstancia personal. Estudios previos señalan que el voluntariado suele mostrar este tipo de motivaciones con cierta frecuencia (Chacón et al., 2010; Omoto y Snyder, 1995). Sin embargo, en los voluntarios on-line aparecieron motivaciones heterocentradas como ayudar a otros, sentimiento de paz o hacer algo útil. Estos resultados coinciden con los encontrados en otros trabajos, que destacan el interés humanitario y el altruismo como una de las principales razones para que los individuos desarrollen actividades de voluntariado (Valor-Segura y RodríguezBailón, 2011). Sin embargo, aunque en nuestro estudio el altruismo aparecía como una de las principales motivaciones para que algunos de los voluntarios se implicaran en este tipo de actividades, hacer algo útil se relacionó con la satisfacción vital sólo para los voluntarios de la prueba en papel para los que además resultaba contraproducente tener un familiar voluntario; mientras que el motivo de petición de la or- 
ganización se vinculó a la satisfacción vital en el voluntariado que rellenó la prueba on-line. Tal y como se señaló anteriormente, posiblemente que la organización cuente con el voluntario supone un reconocimiento social (Cnaan y Cascio, 1998) pero además el individuo puede hacer una valoración positiva de la organización, produciéndose una adecuación recíproca entre la organización y la persona (Thoits y Hewitt, 2001). Una investigación reciente realizada en España muestra que el compromiso de los individuos no sólo explica el compromiso con la organización sino también la satisfacción y el bienestar del voluntariado (Vecina y Chacón, 2013). Además, para los voluntarios que hicieron las pruebas en papel el sentimiento de paz permitía decrecer las emociones negativas y para aquellos que las hicieron on-line eran los estudios afines los que contrarrestaban las emociones negativas, mientras que las experiencias vitales y las circunstancias personales se asociaban positivamente a emociones negativas. Los participantes no detallaron cuáles habían sido dichas circunstancias, pero quizá pudieron ser de valencia negativa y al implicarse en la actividad de voluntariado estén rememorando esa emoción y de ahí la mayor presencia de emociones negativas. En algunos estudios se ha encontrado que los individuos desarrollan conductas de ayuda tras experimentar sentimientos negativos de culpa (Bandura, 1977) o por reducir el malestar que genera observar las necesidades de los otros (Piliavin, Dovidio, Gaertner, y Clark, 1981) parece que para los voluntarios on-line este tipo de motivos relacionados con experiencias previas les impulsan a participar en el voluntariado pero les está generando emociones negativas posiblemente por no tener resueltas determinadas cuestiones vitales.

Por otra parte, aunque la investigación previa apunta la relación entre tiempo dedicado al voluntariado y bienestar (Dávila y Díaz, 2005; Harlow y Cantor, 1996; Jirovec y Hyduk, 1998). En nuestro estudio no aparecieron relaciones significativas del bienestar con el tiempo que llevaban los voluntarios en la organización ni con la frecuencia en que realizaban estas actividades. Tanto los voluntarios que hicieron la prueba en papel como los on-line tenían una gran implicación con el ejercicio del voluntariado estando la mayor parte vinculados a asociaciones desde hacía más de 4 años y dedicando al menos 4 horas semanales a esta actividad. Quizá haber considerado a ambos grupos por separado pudo haber disminuido la variabilidad en el tiempo destinado al voluntariado y de ahí que no se hayan observado diferencias significativas en relación al bienestar.

Las características personales estuvieron más implicadas en el bienestar del voluntariado que las motivaciones, aunque dependía del tipo de voluntariado y del indicador de bienestar considerado. Trabajos recientes señalan que las características personales explican mejor la salud física y mental que la experiencia de voluntariado en si misma (King, Jackson, Morrow-Howell y Oltmanns, 2014; Poulin, 2014). En nuestro estudio, el neuroticismo se relacionó negativamente con todos los indicadores de bienestar en ambos grupos de voluntarios. La investigación previa con población no- voluntaria ha mostrado de forma consistente los efectos negativos del neuroticismo en el bienestar, siendo el predictor más potente (DeNeve y Cooper, 1998; Chico, 2006; Marrero y Carballeira, 2011; Steel et al., 2008). Sin embargo, llama la atención que la extraversión fue el rasgo que más se relacionó con el bienestar de los voluntarios que rellenaron la prueba en papel. Concretamente el gregarismo, la calidez, la actividad, la disciplina, y la ausencia de depresión y hostilidad fueron las facetas que se asociaron en mayor medida al bienestar. En el caso de los voluntarios que hicieron las pruebas on-line, el bienestar se asoció principalmente a la responsabilidad. De las facetas, la más relevante fue la disciplina que explicaba parte de la felicidad pero además la ausencia de depresión, de ansiedad y de sensibilidad a los demás tuvieron un peso importante en el resto de indicadores de bienestar para este grupo de voluntarios. En cuanto a las emociones positivas estuvieron vinculadas a la amabilidad para los voluntarios de la prueba en papel y a la apertura a la experiencia para los on-line. En estudios previos el altruismo, una faceta de la amabilidad, ha mostrado ser una característica de las personas voluntarias (Clary y Miller, 1986; Elshaug y Metzer, 2001; Ruiz de Olabuénaga, 2001) pero no parece que incida en el bienestar al menos de todo el voluntariado. Los voluntarios que realizaron la prueba en papel provenían principalmente de un curso de formación, lo que podría estar explicando los resultados encontrados. Las personas extravertidas se caracterizan por buscar estimulación externa y ser más sensibles a la presión social, además parece que satisfacer la necesidad de hacer algo útil y tener un familiar voluntario les puede estar haciendo más visibles ante los demás, lo que a su vez podría redundar en un mayor reconocimiento social. Por su parte, los voluntarios on-line que presentaron mayor bienestar fueron los que se caracterizaron por una mayor responsabilidad -disciplina- y por una mayor estabilidad emocional, en cierta manera parece que, al contrario que los voluntarios que rellenaron la prueba en papel, el control de la situación se sitúa dentro de ellos mismos en lugar de en el exterior. Hasta el punto que mostraron una menor sensibilidad ante los otros posiblemente como mecanismo para controlar el exceso de implicación que repercutiría negativamente en su bienestar. Algunos estudios que comparan la equivalencia entre las pruebas administradas por internet y en papel encuentran que las personas muestran interés en participar en investigaciones por internet por el efecto de la novedad lo que puede hacer que incrementen inicialmente su nivel de respuesta y omitan en menor medida información recabada mediante estos instrumentos (McDonald y Adam, 2003) pudiendo existir una menor deseabilidad social y una mayor motivación intrínseca en estos voluntarios.

Las emociones positivas y negativas de ambos grupos de voluntarios estaban asociadas en gran medida a la ausencia o presencia de ansiedad y depresión. Estos resultados coinciden con los encontrados en trabajos previos que indican que la práctica del voluntariado se asocia a baja depresión, alta satisfacción vital y felicidad (Thoits y Hewitt, 2001; Li y Fe- 
rraro, 2006; Musick y Wilson, 2003). Además, la edad predecía las emociones positivas y negativas de las personas que cumplimentaron las pruebas en papel, las personas con más edad mostraron más emociones positivas y menos negativas. Estos resultados podrían explicarse en base a la teoría de la selectividad socioemocional (Carstensen, 1995), las personas dependiendo de la edad realizan distintas evaluaciones cognitivas de los acontecimientos según la saliencia de los mismos. De modo que compensan las pérdidas que se producen a nivel físico con una mayor atención a experiencias emocionales significativas. Las personas de más edad suelen informar de escasas experiencias emocionales negativas y mayor control emocional (Gross, Carstensen, Pasupathi, Tsai, Götestam-Skorpen y Hsu, 1997; Mroczek y Kolarz, 1998). Sin embargo, esta relación entre edad y emociones también aparece modulada por diversas variables como la extraversión o el estatus marital (Mroczek, 2001).

En general, se ha identificado un patrón diferencial de voluntariado según sean personas que son más "visibles" o "invisibles". Las personas que cumplimentaron las pruebas en papel que mostraron mayor bienestar fueron más extravertidas, más gregarias y estaban más implicadas en actividades formativas, estaban motivadas por hacer algo útil para los demás y por desarrollar su autorrealización; mientras que el bienestar de las personas que hicieron las pruebas on-line dependió principalmente de la disciplina, de la petición de la organización y de circunstancias personales. Thoits y Hewitt (2001) ya apuntaban que existen factores determinantes, tanto recursos personales como salud física y mental, que inciden en los procesos de autoselección y de selección social del voluntariado, por lo que habría que profundizar acerca

\section{Referencias}

Allen, N. J., \& Rushton, J. P. (1983). Personality characteristics of community mental health volunteers: A review. Journal of Voluntary Action Research, 12, 36-49. doi: 10.1177/089976408301200106

Atienza, F. L., Pons, D., Balaguer, I., \& García-Merita, M. (2000). Propiedades psicométricas de la Escala de Satisfacción con la Vida en adolescentes. Psicothema, 12, 314-319. Recuperado de http://www.unioviedo.net/reunido/index.php/PST/article/view/7597

Babchuk, N., \& Gordon, C. W. (1962). The voluntary association in the slum. Lincoln, NB: University of Nebraska.

Bandura, A. (1977). Social learning theory. Englewood Cliffs, NJ: Prentice-Hall.

Benson, P. L., Dehority, J., Garman, L., Hanson, E., Lebold, C., Hochschwender, M., Rohr, R., \& Sullivan, J. (1980). Intrapersonal correlates of non-spontaneaus helping behaviour. The Journal of Social Psychology, 110, 87-95. doi: 10.1080/00224545.1980.9924226.

Bradburn, N. M. (1969). The structure of psychological well-being. Chicago: Aldine.

Buchanan, T., Ali, T., Heffernan, T. M., Ling, J., Parrott, A. C., Rodgers, J., \& Scholey, A. B. (2005). Nonequivalence of on-line and paper-andpencil psychological tests: The case of the prospective memory questionnaire. Behavior Research Methods, 37, 148-154. doi: 10.3758/BF03206409

Carstensen, L. L. (1995). Evidence for a life-span theory of socioemotional selectivity. Current Directions in Psychological Science, 151-156. Recuperado de http://www.jstor.org/stable/20182356

Chacón, F. (2005) ¿Influye el burnout en la intención de continuar de voluntarios en actividades de bajo nivel de estrés? Cuadernos de Trabajo Social, 17, 117-130. Recuperado de de si esas características hacen que el voluntariado se implique en distintos tipos de actividades y/o en distintos formatos de participación. También, hay que tener en cuenta que las muestras que participan en investigaciones a través de internet suelen ser más heterogéneas, por lo que podría haber más variación en los constructos latentes evaluados (Buchanan et al., 2005). En el futuro habría que seguir indagando si las diferencias que han aparecido entre ambos grupos de voluntarios podrían responder a diferentes estructuras de los cuestionarios empleados o a distinto perfil del voluntariado. Además, hay que tener en cuenta que los participantes de este estudio han sido principalmente adultos-jóvenes y mayoritariamente mujeres, por lo que los resultados no deben ser generalizados a otros grupos de voluntarios. Trabajos previos ya han señalado diferencias entre el voluntariado en base a su edad (Van Willigen, 2000; Yubero y Larrañaga, 2002). En nuestro estudio apenas participaron personas de edad avanzada por lo que no fue posible analizar en profundidad si los motivos o características personales que subyacían a la participación voluntaria diferían en los distintos períodos evolutivos, aunque se ha observado algún efecto de la edad en las emociones positivas y negativas. Por otra parte podrían existir distintos perfiles de voluntarios en función del tipo de actividad que lleven a cabo (Wilson, 2000), aspecto que no pudo ser analizado debido al tamaño de la muestra. En el futuro habría que continuar investigando acerca de si existe un patrón de personalidad específico del voluntario en relación a personas que no realizan estas actividades y cuáles de estas características personales redundan en un mayor bienestar.

http://revistas.ucm.es/index.php/CUTS/article/view/CUTS04041101 $17 \mathrm{~A} / 7610$

Chacón, F., Pérez, T., Flores, J. y Vecina, M.L. (2010). Motivos del voluntariado: categorización de las motivaciones de los voluntariados mediante pregunta abierta. Intervención psicosocial: Revista sobre igualdad y calidad de vida, 19, 213-222. doi: 10.5093/in2010v19n3a2

Chacón, F., \& Vecina, M. L. (1999). Motivaciones y burnout en el voluntariado. Psychosocial Intervention, 8, 31-42. Recuperado de http://www.copmadrid.org/webcopm/publicaciones/social/47560.pdf

Chico, E. (2006). Personality dimensions and subjective well-being. The Spanish Journal of Psychology, 9, 38-44. Recuperado de http://revistas.ucm.es/index.php/SJOP/article/viewFile/SJOP060612 0038A/29060

CIS (2011). Estudio n ${ }^{\circ} 2.864$. Recuperado de http://datos.cis.es/pdf/Es2864cs_A.pdf

Clary, E. G., \& Miller, J. (1986). Socialization and situational influences on sustained altruism. Child Development, 57, 1358-1369. Recuperado de http://www.jstor.org/stable/1130415

Clary, E. C., Snyder, M., Ridge, R.D., Copeland, J., Stukas, A. A., Hauger, J., \& Miene, P. (1998). Understanding and assessing the motivations of volunteers: a functional approach. Journal of Personality and Social Psychology, $74, \quad 1516-1530 . \quad$ Recuperado de http://www.comm.umn.edu/ akoerner/courses/5431S13/Clary\%20et\%20al.\%20(1998).pdf

Cnaan, R. A., \& Cascio, T. A. (1998). Performance and commitment: Issues in management of volunteers in human service organizations. Journal of Social Service Research, 24, 1-37. Recuperado de 
http:/ / repository.upenn.edu/cgi/viewcontent.cgi?article=1000\&contex $\mathrm{t}=$ spp_papers\&sei-

re-

dir $=1 \&$ referer $=$ http $\% 3 \mathrm{~A} \% 2 \mathrm{~F} \% 2 \mathrm{Fscholar}$.google.es $\% 2 \mathrm{Fscholar} \% 3 \mathrm{Fq} \%$ 3DPerformance $\% 2$ Band $\% 2$ Bcommitment $\% 253 \mathrm{~A} \% 2 \mathrm{BIssues} \% 2 \mathrm{Bin} \% 2$ Bmanage-

ment $\% 2 \mathrm{Bof} \% 2 \mathrm{Bvolunteers} \% 2 \mathrm{Bin} \% 2 \mathrm{Bhuman} \% 2 \mathrm{Bservice} \% 2 \mathrm{~B}$ organiza tions $\% 26 \mathrm{btnG} \% 3 \mathrm{D} \% 26 \mathrm{hl} \% 3 \mathrm{Des} \% 26$ as sdt $\% 3 \mathrm{D} 0 \% 252 \mathrm{C} 5 \#$ search $=\%$ 22 Performance $\% 20$ commitment $\% 3 \mathrm{~A} \% 20$ Issues $\% 20$ management $\% 20 \mathrm{v}$ olunteers $\% 20$ human $\% 20$ service $\% 20$ organizations $\% 22$

Cordero, A., Pamos, A. y Seisdedos, N. (1999). Inventario de Personalidad NEO Revisado (NEO PI-R) e Inventario NEO Reducido de Cinco Factores (NEOFFI). Manual profesional. Madrid: TEA Ediciones, S.A.

Costa, P. T, \& McCrae, R. R. (1992). Revised NEO Personality Inventory (NEOPI-R) and NEO Five-Factor Inventory (NEO-FFI) professional manual. Odessa, FL: Psychological Assessment Resources.

Dávila, M. C. (2003). La incidencia diferencial de los factores psicosociales en distintos tipos de voluntariado. Tesis doctoral no publicada, Universidad Complutense de Madrid, Madrid. Recuperado de http://biblioteca.ucm.es/tesis/psi/ucm-t26478.pdf

Dávila. M. C. y Díaz, J. F. (2005). Voluntariado y satisfacción vital. Intervención Psicosocial, 14, 81-84. Recuperado de http://www.copmadrid.org/webcopm/publicaciones/social/94939.pdf

DeNeve, K. M., \& Cooper, H. (1998). The happy personality: A metaanalysis of 137 personality traits and subjective well-being. Psychological Bulletin, 124, 197-229. doi: 10.1037/0033-2909.124.2.197

Diener, E. (1984). Subjective well-being. Psychological Bulletin, 95, 542-575. doi: 10.1037/0033-2909.95.3.542

Diener, E., Emmons, R. A., Larsen, R. J., \& Griffin, S. (1985). The Satisfaction with Life Scale. Journal of Personality Assessment, 49, 71-75. doi: 10.1207/s15327752jpa4901_13

Diener, E., Suh, E. M., Lucas, R. E., \& Smith, H. L. (1999). Subjective wellbeing. Three decades of progress. Psychological Bulletin, 125, 276 -302. doi: 10.1037/0033-2909.125.2.276

Dulin, P., Hill, R. D., \& Anderson, J. (2001). Altruism as a predictor of life satisfaction in older low income service providers. Journal of Mental Health \& Aging, 4, 349-359.

Eisenberg, N., \& Miller, P. A. (1987). The relation of empathy to prosocial and related behaviors. Psychological Bulletin, 101, 91- 119. doi: 10.1037/0033-2909.101.1.91

Elshaug, C., \& Metzer, J. (2001). Personality attributes of volunteers and paid workers engaged in similar occupational tasks. The Journal of Social Psychology, 141, 752-763. doi: 10.1080/00224540109600586

Extremera, N., Salguero, J. M., \& Fernández-Berrocal, P. (2011). Trait metamood and subjective happiness: A 7-week prospective study. Journal of Happiness Studies, 12, 509-517. doi: 10.1007/s10902-010-9233-7

FEAPS (2011). El voluntariado en el movimiento asociativo. Recuperado de http://www.feaps.org/component/content/article/783-iii-encuestade-voluntariado-feaps-un-voluntariado-mas-diverso-inclusivo-ycentrado-en-apoyos-individuales.html

GHK (2010). Study on Volunteering in the European Union. Bruselas: Educational, Audiovisual \& Culture Executive Agency (EAC-EA). Directorate General Education and Culture (DG-EAC). Recuperado de http://ec.europa.eu/citizenship/pdf/doc1018_en.pdf

Gross, J. J., Carstensen, L. L., Pasupathi, M., Tsai, J., Götestam-Skorpen, C., \& Hsu, A. Y. (1997). Emotion and aging: experience, expression, and control. Psychology and Aging, 12, 590-599. doi: 10.1037/08827974.12.4.590

Harlow, R. E., \& Cantor, N. (1996). Still participating after all these years: A study of life task participation in later life. Journal of Personality and Social Psychology, 71, 1235-1249. doi: 10.1037/0022-3514.71.6.1235

Herman, K., \& Usita, P. M. (1994). Predicting Big Brothers/Big Sisters volunteer attrition with 16 PF. Child and Youth Care Forum, 23, 207-211. Recuperado de http://link.springer.com/article/10.1007/BF02209229

Hettman, D. W., \& Jenkins, E. (1990). Volunteerism and social interest. Individual Psychology: Journal of Adlerian Theory, Research \& Practice, 46, 298-303.

Ho, Y. W., You, J., \& Fung, H. H. (2012). The moderating role of age in the relationship between volunteering motives and well-being. European Journal of Ageing, 9, 319-327. doi: 10.1007/s10433-012-0245-5
INE (2007). Participación en actividades de organizaciones benéficas y voluntariado informal. Recuperado de http://www.ine.es/jaxi/tabla.do

Instituto de la Mujer (2013). Mujeres en cifras. Boletín digital estadístico, 2, 1-2. Recuperado

http://www.inmujer.gob.es/estadisticas/boletinEstadistico/docs/num ero_2_Febrero_2013.pdf

Janoski, T., Musick, M., \& Wilson, J. (1998). Being volunteered? The impact of social participation and pro-social attitudes on volunteering. Sociological Forum, 13, 495-519. Recuperado de http://deepblue.lib.umich.edu/bitstream/handle/2027.42/45653/1120 6_2004_Article_415800.pdf?sequence=1

Jirovec, R. L., \& Hyduk, C. A. (1998). Type of volunteer experience and health among older adult volunteers. Journal of Gerontological Social Work, 30, 29-42. doi: 10.1300/J083v30n03_04

Juventud en Acción (2014). Recursos formativos para voluntarios y voluntarias del SVE. Recuperado http://www.formacionsve.es/salida/5_motivacion.php

King, H. R., Jackson, J. J., Morrow-Howell, N., \& Oltmanns, T. F. (2014). Personality Accounts for the Connection Between Volunteering and Health. The Journals of Gerontology Series B: Psychological Sciences and Social Sciences. doi: 10.1093/geronb/gbu012

Kwok, Y. Y., Chui, W. H., \& Wong, L. P. (2012) Need Satisfaction Mechanism Linking Volunteer Motivation and Life Satisfaction: A Mediation Study of Volunteers Subjective Well-Being. Social Indicators Research, 1, 1 15. doi: 10.1007/s11205-012-0204-8

Latané, B., \& Darley, J. M. (1970). The unresponsive bystander: Why doesn't he belp? New York: Appleton- Crofts.

Ley Orgánica 6/1996, de 15 de enero, del. Voluntariado. BOE, 17 de enero de 1996, núm.15, p. 1239-1243. Recuperado de http://www.boe.es/boe/dias/1996/01/17/pdfs/A01239-01243.pdf

Li, Y., \& Ferraro, K. F. (2006). Volunteering in middle and later life: Is health a benefit, barrier or both? Social Forces, 85, 497-519. doi: $10.1353 /$ sof.2006.0132

Lyubomirsky, S., \& Lepper, H. S. (1999). A measure of subjective happiness: Preliminary reliability and construct validation. Social Indicators Research, 46, 137-155. Recuperado de http://my.psychologytoday.com/files/attachments/496/subjectivehappiness-scale.pdf

Marrero, R. J. y Carballeira, M. (2011). Well-being and personality: facet-level analyses. Journal of Personality and Individual Differences, 50, 206-21. doi: 10.1016/j.paid.2010.09.030

Marrero, R. J., Carballeira, M. y Rodríguez, J. P. (2007). El papel del optimismo en la salud. Memoria de investigación no publicada, Universidad de La Laguna, La Laguna.

McDonald, H., \& Adam, S. (2003). A comparison of online and postal data collection methods in marketing research. Marketing Intelligence \& Planning, 21, 85-95. doi: 10.1108/02634500310465399

Meier, S., \& Stutzer, A. (2008). Is volunteering rewarding in itself? Economica, 75, 39-59. doi: 10.1111/j.1468-0335.2007.00597.x

Metzer, J. C. (2003). Volunteer Work: Stress and satisfaction at the turn of the 21st Century. En F. R. Dollard, A. H. Winefield y H. R. Winefield (Eds.), Psychological Stress in the Human Service Professions. London: Taylor \& Francis. Recuperado de http://books.google.es/books?hl=es\&lr=\&id=_br8RhFNGugC\&oi=f nd\&pg=PA389\&dq=Metzer, + J.C. $+(2003) .+$ Volunteer + Work: + Stress + and + satisfaction + at + the + turn + of + the +21 st + Century.\&ots $=$ B_Aai$\mathrm{k} \_3 \mathrm{~d} \& s i g=76 \mathrm{bX} 1 \mathrm{EM} 80 \_9 \mathrm{BcPa}-\mathrm{r} 765 \mathrm{Qd} 9 \mathrm{gVr} 0 \# \mathrm{v}=$ onepage\&q\&f=false

Mroczek, D. K. (2001). Age and emotion in adulthood. Current Directions in Psychological Science, 10, 87-90. doi: 10.1111/1467-8721.00122

Mroczek, D. K., \& Kolarz, C. M. (1998). The effect of age on positive and negative affect: a developmental perspective on happiness. Journal of Personality and Social Psychology, 75, 1333-1349. doi: 10.1037/00223514.75.5.1333

Myers, S. A. (1995). Student perceptions of teacher affinity-seeking and classroom climate. Communication Research Reports, 12, 192-199. doi: $10.1080 / 08824099509362056$

Musick, M. A., \& Wilson, J. (2003). Volunteering and depression: The role of psychological and social resources in different age groups. Social Science \& Medicine, 56, 259-269. doi: 10.1016/S0277-9536(02)00025-4 
Okun, M. A., \& Schultz, A. (2003). Age and motives for volunteering: testing hypotheses derived from socioemotional selectivity theory. Psychology and Aging, 18, 231-239. doi: 10.1037/0882-7974.18.2.231

Omoto, A. M., \& Snyder, M. (1995). Sustained helping without obligation: motivation, longevity of service, and perceived attitude change among AIDS volunteers. Journal of Personality and Social Psychology, 68, 671-686. doi: 10.1037/0022-3514.68.4.671

Omoto, A. M., Snyder, M., \& Martino, S. C. (2000). Volunteerism and the life course: Investigating age-related agendas for action. Basic and Applied Social Psychology, 22, 181-197. doi: 10.1207/S15324834BASP2203 6

Penner, L. A. (2002). Dispositional and organizational influences on sustained volunteerism: An interactionist perspective. Journal of Social Issues, 58, 447-467. doi: 10.1111/1540-4560.00270

Plataforma de Voluntariado de España (2011). Diagnóstico de la situación del voluntariado de acción social en España. Informes, estudios e investigación. Madrid: Ministerio de Sanidad, Política Social e Igualdad. Recuperado de file: ///C:/Users/optimismo/Downloads/diagnostico_situacion_voluntari ado.pdf

Plataforma de Voluntariado de España (2013). Asi somos: El perfil del voluntariado social en España. Madrid: Ministerio de Sanidad, Política Social e Igualdad. Recuperado http://www.plataformavoluntariado.org/ARCHIVO/documentos/rec ursos/ASI_SOMOS.pdf

Pierrucci, J., \& Noel, R. C. (1980). Duration of participation of correctional volunteers as a function of personal and situational variables. Journal of Community Psychology, $8, \quad 245-250$. doi: 10.1002/15206629(198007)8:3<245::AID-JCOP2290080308>3.0.CO;2-U

Piliavin, J. A., Dovidio, J. F., Gaertner, S. L., \& Clark, R. D. (1981). Emergency intervention. New York: Academic Press.

Plagnol, A. C., \& Huppert, F. A. (2010). Happy to help? Exploring the factors associated with variations in rates of volunteering across Europe. Social Indicators Research, 97, 157-176. doi: 10.1007/s11205-0099494-x

Poulin, M. J. (2014). Volunteering predicts health among those who value others: Two national studies. Health Psychology, 33, 120-129. doi: $10.1037 / \mathrm{a} 0031620$

Pushkar, D., Reis, M., \& Morros, M. (2002). Motivation, personality and well-being in older volunteers. The International Journal of Aging and Human Development, 55, 141-162. doi: 10.2190/MR79-J7JA-CCX5-U4GQ

Ruiz de Olabuénaga, J. I. (2001). El sector no lucrativo en España. CIRIECEspaña, Revista de Economía Pública, Social y Cooperativa, 37, 51-78. Recuperado de http://www.ciriec-revistaeconomia.es/banco/03_Ruiz_37.pdf

Sandín, B., Chorot, P., Lostao, L., Joiner, T. E., Santed, M. A., \& Valiente, R. M. (1999). Escalas PANAS de afecto positivo y negativo: validación factorial y convergencia transcultural. Psicothema, 11(1), 37-51. Recuperado http://www.unioviedo.net/reunido/index.php/PST/article/view/7556

Smith, D. H. (1994). Determinants of voluntary association participation and volunteering: a literature review. Nonprofit and Voluntary Sector Quarterly, 23, 243-263. doi: 10.1177/089976409402300305
Spitz, R.T., \& MacKinnon, J. R. (1993). Predicting success in volunteer community service. Psychological Reports, 73, 815-818. doi: 10.2466/pr0.1993.73.3.815

Steel, P., Schmidt, J., \& Shultz, J. (2008). Refining the relationship between personality and subjective well-being. Psychological Bulletin, 134, 138-161. doi: 10.1037/0033-2909.134.1.138

Thoits, P. A., \& Hewitt, L. N. (2001). Volunteer work and well-being. Journal of Health and Social Behavior, 42, 115-131. Recuperado de http://wwvg.asanet.org/images/members/docs/pdf/featured/volunte er.pdf

Unger, L. S., \& Thumuluri, L. K. (1997). Trait empathy and continuous helping: The case of voluntarism. Journal of Social Behaviour \& Personality, 12, 785-800.

Valor-Segura, I. y Rodríguez-Bailón, R. (2011). Motivaciones, compromiso y experiencia voluntaria en estudiantes universitarios españoles. Anales de Psicología, 27, 157-164. Recuperado de http://revistas.um.es/analesps/article/view/113571/107561

Van Willigen, M. (2000). Differential benefits of volunteering across the life course. The Journals of Gerontology Series B: Psychological Sciences and Social Sciences, 55, S308-S318. doi: 10.1093/geronb/55.5.S308

Vázquez, C., Duque, A., \& Hervás, G. (2013). Satisfaction With Life Scale in a representative sample of Spanish adults: Validation and normative data. Spanish Journal of Psychology, 16, 1-15. doi:10.1017/sjp.2013.82

Vecina, M. L. y Chacón, F. (2013). ¿Es el engagement diferente de la satisfacción y del compromiso organizacional? Relaciones con la intención de permanencia, el bienestar psicológico y la salud física percibida en voluntarios. Anales de Psicologia, 29, 225-232. doi: http://dx.doi.org/10.6018/analesps.29.1.161861

Wandersman, A., Florin, P., Friedmann, R. R., \& Meier, R. (1987). Who participates, who does not participe, and why? Participation in block and neighborhood organizations in the United States and Israel. Sociological Forum, 2, 534-555. doi: 10.1007/BF01106625

Watson, D., Clark, L. A., \& Tellegen, A. (1988). Development and validation of brief measures of positive and negative affect: the PANAS scales. Journal of Personality and Social Psychology, 54, 1063-1070. doi: 10.1037/0022-3514.54.6.1063

Wheeler, J. Gorey, K., \& Greenblatt, B. (1998). The Beneficial Effects of Volunteering for Older Adults and the People They Serve: A Metaanalysis. International Journal of Aging and Human Development, 47, 69-80. doi: 10.2190/VUMP-XCMF-FQYU-VOJH

Wilson, J. (2000). Volunteering. Annual Review of Sociology, 26, 215-240. Recuperado de http://www.jstor.org/stable/223443

Yubero, S. y Larrañaga, M. E. (2002). Concepción del voluntariado desde la perspectiva motivacional: conducta de ayuda vs. altruismo. Pedagogía Social: Revista Interuniversitaria, 9, 27-39. Recuperado de http:/ / dialnet.unirioja.es/servlet/articulo? codigo $=994918$

(Artículo recibido: 05-08-2013; revisado: 04-04-2014; aceptado: 25-06-2014) 